\title{
TEM Study of Amorphous Carbon with Fully sp3-Bonded Structure
}

Jianguo Wen ${ }^{1}$, Zhidan Zeng ${ }^{2}$, Liuxiang Yang ${ }^{2,3}$, Qiaoshi Zeng ${ }^{2}$, Hongbo Lou ${ }^{2}$, Hongwei Sheng $^{2}$, Dean J. Miller ${ }^{1}$, Wenge Yang ${ }^{2,3}$ Ho-kwang $\mathrm{Mao}^{2,3}$

${ }^{1}$ Center for Nanoscale Materials, Argonne National Laboratory, Argonne, IL 60439

${ }^{2}$ Center for High Pressure Science and Technology Advanced Research (HPSTAR), Shanghai 201203, China

${ }^{3}$ HPSynC, Geophysical Laboratory, Carnegie Institution of Washington, Argonne, IL 60439.

In 2011, Lin etal found that glassy carbon was converted into a new carbon allotrope with a fully sp3-bonded amorphous structure under high pressure of about $45 \mathrm{GPa}$ [1]. However, the transition was reversible upon releasing pressure. Recently, Zeng etal synthesized quenchable amorphous diamond from glassy carbon with the combination of high pressure and in situ laser heating [2]. To understand the atomic structure and chemical bonding, we studied the recovered carbon materials using aberration corrected TEM and electron energy-loss spectroscopy (EELS). We confirmed that the recovered material is amorphous diamond with completely tetrahedral sp3-bonds.

We first investigated the structure and chemical bonding of the starting material. Glassy carbon (Fig. 1b) consists of numerous curved graphitic layers with interlayer distances ranging from 3.53.9 A. For comparison, we also studied curved graphitic carbon and amorphous carbon with different degree of crystallinity of graphitic carbon as shown in HREM images in Fig. 1. With the aberration-corrected TEM, some fragmented curved graphene can be observed in the amorphous carbon (Fig. 1c). EELS spectra of glassy carbon show a strong pre-peak at $\sim 285 \mathrm{eV}$, corresponding to the transitions of 1 s electrons to $\pi *$ state, as a result of its nearly $100 \% \operatorname{sp} 2$ bonds. With the decrease of crystallinity, the pre-peak intensity decreases, indicating an increase in the degree of $\mathrm{sp} 3$ bonding. In addition to the difference in pre-peak, the fine structures corresponding to $\sigma$ bonding become vague in amorphous carbon.

HREM images show that the recovered carbon does not have a layered structure (Fig. 2b). The randomly arranged atoms in the HRTEM image and diffuse diffraction rings in selected-area electron diffraction (SAD) both indicate that the recovered sample has no identifiable crystalline symmetry. Nanocrystalline diamond with crystal size of 1-3 nm shows obvious diamond atomic arrangements in Fig. 2a and sharp diffraction rings in SAD pattern. The carbon K-edge EELS of the recovered sample shows no pre-peak, the same as for the nanocrystalline diamond, indicating both are dominated by sp3 bonds. Compared with the nanocrystalline diamond, the fine structures in amorphous diamond also become vague. Using the structural model of the tetrahedral amorphous carbon, we found the calculated EELS based on this structural model shows the same features as the experimental EELS of amorphous diamond.

Although measurement of density is difficult for the high pressure samples due to the small size of the sample, we were able to estimate the density of these samples based on their plasmon peak energy derived from low loss EELS. The plasmon peak for the amorphous diamond is at 31.8 $\mathrm{eV}$. From the peak position, we estimated the density of the recovered sample to be $3.3 \pm 0.1$ $\mathrm{g} / \mathrm{cm}^{3}$, which is close to the density of diamond $\left(3.52 \mathrm{~g} / \mathrm{cm}^{3}\right)$. 


\section{References:}

[1] Y. Lin, L. Zhang, H.-K. Mao, P. Chow, Y. Xiao, M. Baldini, J. Shu, and W. L. Mao. Phys. Rev. Lett. 107, 175504 (2011).

[2] Z. Zeng, L. Yang, Q. Zeng, H. Lou, H. Sheng, J. Wen, D.J. Miller, Y. Meng, W. Yang, W.L. Mao, H.-K. Mao, Nature Communication (in press).

[4] Electron microscopy was performed at the Center for Nanoscale Materials at Argonne National Laboratory, a U.S. Department of Energy Office of Science Laboratory operated under Contract No. DE-AC02-06CH11357 by UChicago Argonne, LLC. The authors acknowledge the financial support of the National Natural Science Foundation of China (grant U1530402)
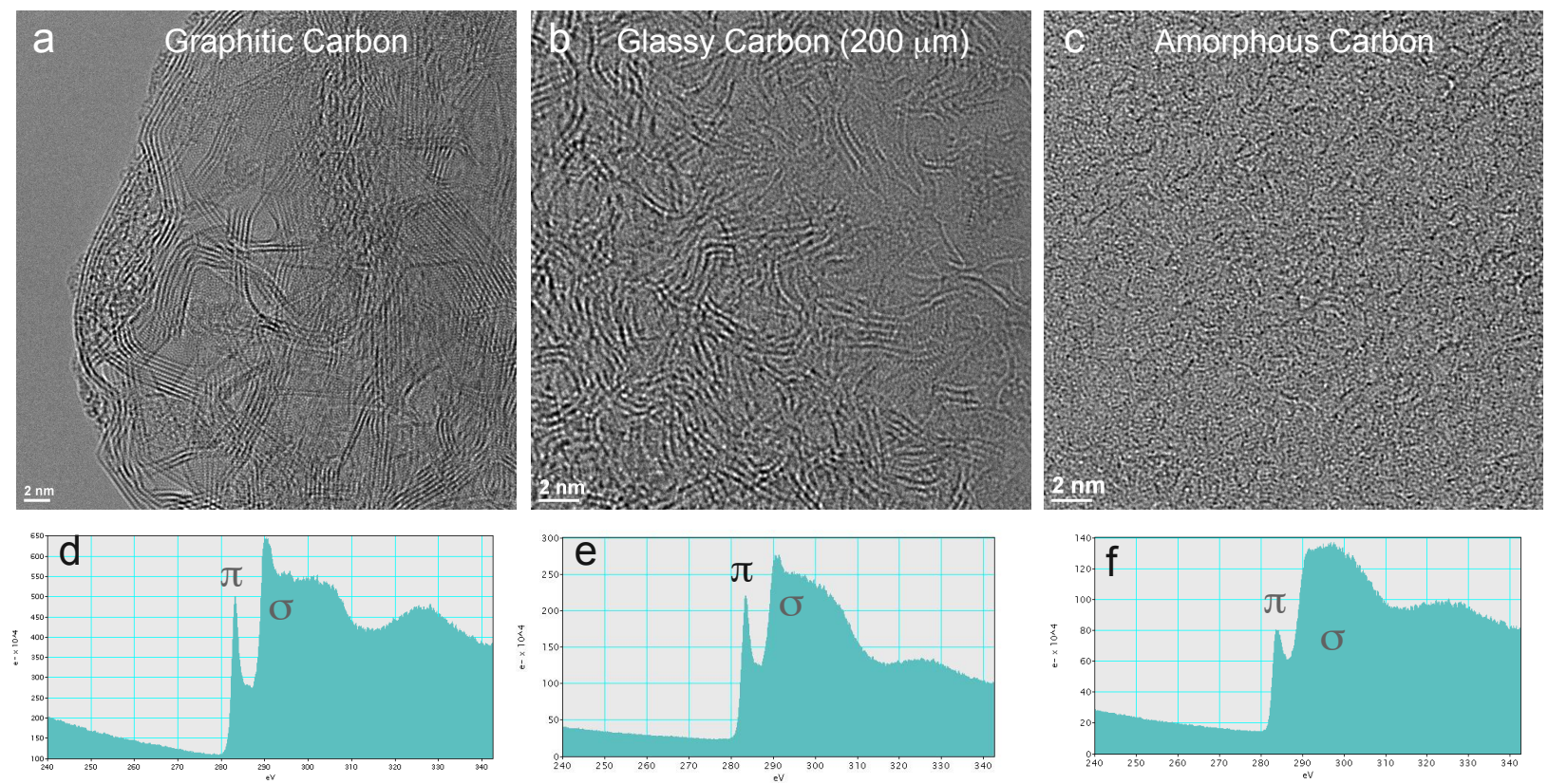

Fig. 1. HREM images of (a) curved graphitic carbon (b) glass carbon, and (c) amorphous carbon and their corresponding EELS spectra. Pre-peak intensity decreases with the decrease in crystallinity of graphitic carbon.
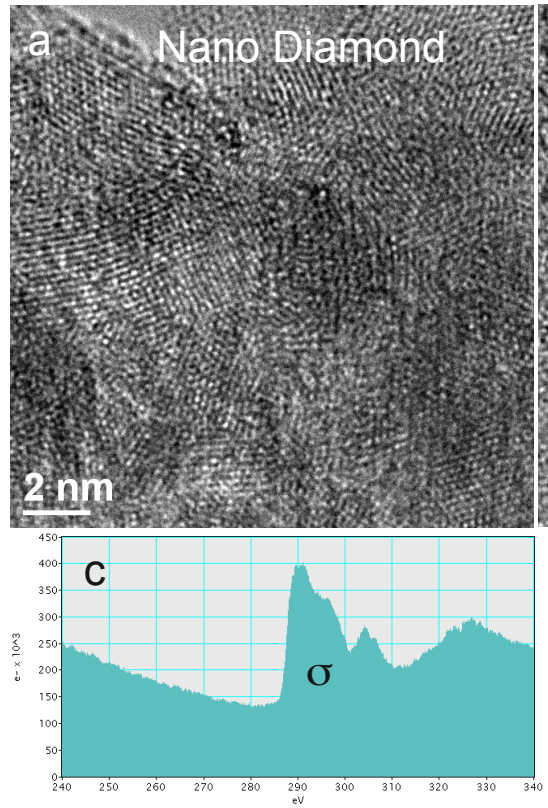
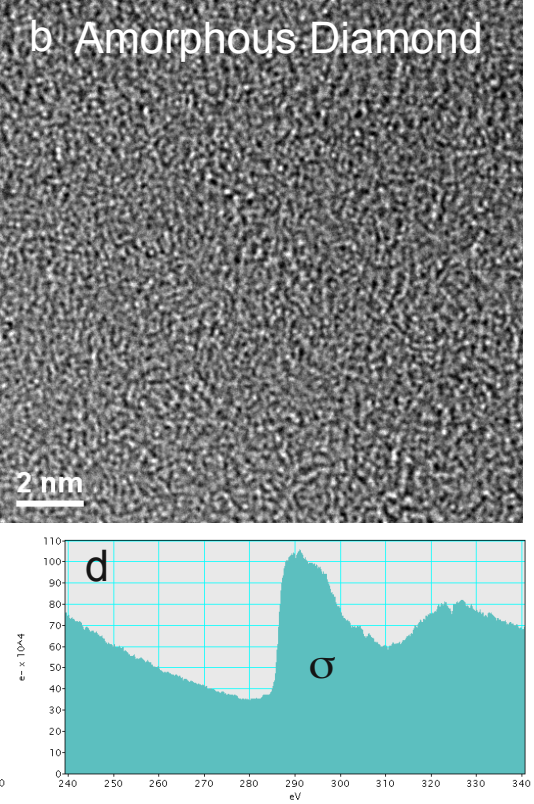

Fig. 2. HREM images of (a) nanodiamond and (b) amorphous diamond. Both EELS spectra show no pre-peak. Fine structures in the EELS spectrum of amorphous become vague, indicating an amorphous nature of this carbon. 